Full Length Article

\title{
Establishment of a bleeding score as a diagnostic tool for patients with rare bleeding disorders
}

\author{
Roberta Palla $^{\mathrm{a}, *}$, Simona M. Siboni ${ }^{\mathrm{b}}$, Marzia Menegatti ${ }^{\mathrm{a}}$, Khaled M Musallam ${ }^{\mathrm{b}}$, \\ Flora Peyvandi ${ }^{\mathrm{a}, \mathrm{b}}$, on behalf of the European Network of Rare Bleeding Disorders (EN-RBD) group \\ a Department of Pathophysiology and Transplantation, Università degli Studi di Milano, and Luigi Villa Foundation, Milan, Italy \\ ${ }^{\mathrm{b}}$ Angelo Bianchi Bonomi Hemophilia and Thrombosis Centre, Fondazione IRCCS Ca' Granda Ospedale Maggiore Policlinico, Milan, Italy
}

\section{A R T I C L E I N F O}

\section{Article history:}

Received 31 August 2016

Received in revised form 3 November 2016

Accepted 9 November 2016

Available online 15 November 2016

\section{Keywords:}

Bleeding score

Diagnostic tool

Rare bleeding disorders

Coagulation factors deficiency

\begin{abstract}
A B S T R A C T
Introduction: Bleeding manifestations among patients with rare bleeding disorders (RBDs) vary significantly between disorders and patients, even when affected with the same disorder. In response to the challenge represented by the clinical assessment of the presence and severity of bleeding symptoms, a number of bleeding score systems (BSSs) or bleeding assessment tools (BATs) were developed. The majority of these were specifically developed for patients with more common bleeding disorders than RBDs. Few RBDs patients were evaluated with these tools and without conclusive results.

Methods: A new BSS was developed using data retrieved from a large group of patients with RBDs enrolled in the EN-RBD database and from healthy subjects. These data included previous bleeding symptoms, frequency, spontaneity, extent, localization, and relationship to prophylaxis and acute treatment. The predictive power of this BSS was also compared with the ISTH-BAT and examined for the severity of RBDs based on coagulant factor activity.

Results: This BSS was able to differentiate patients with RBDs from healthy individuals with a bleeding score value of 1.5 having the highest sum of sensitivity $(67.1 \%)$ and specificity $(73.8 \%)$ in discriminating patients with RBD from those without. An easy-to-use calculation was also developed to assess the probability of having a RBD. Its comparison with the ISTH-BAT confirmed its utility. Finally, in RBDs patients, there was a significant negative correlation between BS and coagulant factor activity level, which was strongest for fibrinogen and FXIII deficiencies.

Conclusion: The use of this quantitative method may represent a valuable support tool to clinicians.
\end{abstract}

(c) 2016 Elsevier Ltd. All rights reserved.

\section{Introduction}

Rare bleeding disorders (RBDs) represent $3-5 \%$ of all inherited coagulation deficiencies and include fibrinogen, factor (F) II, FV, combined FV and FVIII (FV + VIII), FVII, FX, FXI, and FXIII deficiencies [1].

RBDs are characterized by a wide variety of symptoms ranging from mild to severe, which can vary significantly from one disorder to another and from one patient to another with the same type of disorder. The association between the level of factor in plasma and bleeding tendency can also vary markedly between deficiencies and between patients affected with the same deficiency (e.g. FV, FVII and FXI deficiencies), as

Abbreviations: AUC, area under the curve; BAT, bleeding assessment tool; BS, bleeding score; BSS, bleeding score system; CI, 95\% confidence intervals; CNS, central nervous system; EN-RBD, European Network of Rare Bleeding Disorders; F, factor; RBD, rare bleeding disorder; ROC, receiver-operator curve; VWD, von Willebrand disease.

* Corresponding author at: Angelo Bianchi Bonomi Haemophilia and Thrombosis Centre, Via Pace 9, 20122 Milan, Italy.

E-mail address: roberta.palla@unimi.it (R. Palla). recently shown by the European Network of Rare Bleeding Disorders (EN-RBD) study group [2]. Nonetheless, bleeding symptoms are also frequently reported by normal healthy subjects, with at least one episode in up to $25 \%$ of the general population [3,4] with the most commonly reported bleeding symptoms being gum bleeding, epistaxis, minor wounds bleeding and menorrhagia in females (average frequency $28,23,20$ and $35 \%$, respectively).

The clinical appreciation of the presence and severity of hemorrhagic symptoms is an important step in the evaluation of subjects referred for a possible bleeding disorder, including RBDs. However, the evaluation of bleeding symptoms is a challenge because the reporting and interpretation of bleeding symptoms are prone to subjectivity. Significant symptoms may be overlooked because they are considered normal and minimal by affected patients used to have bleeding episodes, or on the contrary, trivial symptoms may be given undue consideration by healthy subjects. Over the years, in response to these challenges, multiple investigators developed a number of bleeding score system (BSSs) or bleeding assessment tools (BATs) in the attempt to standardize 
bleeding histories by identifying questions that best distinguish between patients affected with a bleeding disorder and unaffected individuals [5-10].

Until now, few patients with RBDs were studied using these tools with no conclusive results [11-13]. Two recent reports specifically focused on women with bleeding disorders including RBDs showing that women with RBDs [11] and in particularly with FVII deficiency [12] had a higher prevalence of excessive bleeding at menarche as well as menorrhagia and general bleeding symptoms. Additionally, Siboni et al. [11] showed that in affected women the Sramek bleeding score [6] increased with increasing severity of the coagulation factor defect, although these results are very likely affected by the inclusion of women with von Willebrand disease (VWD) and carriers of hemophilia. Recently, Shapiro and colleagues [13] showed that the bleeding scores, evaluated using the ISTH-BAT [10] did not differ between 35 patients with hereditary dysfibrinogenemia and matched healthy controls. Diagnosis of patients with RBDs has been also performed using the Condensed MCMDM1-VWD Bleeding Questionnaire [9]. Tosetto and colleagues evaluated the diagnostic utility of the Condensed MCMDM1VWD Bleeding Questionnaire in 215 subjects referred for a possible bleeding disorder [14]. The performance of the BAT varied widely depending on the specific reason for referral and 18 out of 215 enrolled subjects were diagnosed with FXI deficiency [14]. Azzam and colleagues described also the diagnostic utility of the Condensed MCMDM-1VWD Bleeding Questionnaire to predict the presence of a bleeding disorder in 30 women with unexplained menorrhagia [15], showing that a high proportion of women enrolled (20/30 or $66.6 \%$ ) had an underlying bleeding disorder, but only three patients had a RBD (one each with fibrinogen, FV, and FV + VIII deficiencies) making it impossible to generalize the results to all RBDs.

The described situation emphasizes the need of an ideal scoring system to be applied for the identification of patients with RBDs. Many of the already developed tools were originally designed for more common bleeding disorders, such as VWD, and it is questionable whether they provide the optimal assessment for patients affected with RBDs.

In this manuscript, we provide a novel tool based on a bleeding score (BS) constructed by retrieving data related to previous bleeding types, frequency, spontaneity, extent, localization, and relationship with prophylaxis and treatment of almost 500 patients enrolled in the EN-RBD database, the largest performed study on RBDs so far [2]. The predictive power of our BSS was also compared with the ISTH-BAT [10] in a subgroup of Italian patients and controls. The ability of the BSS to predict the severity of RBDs based on coagulant factor activity was also examined.

\section{Patients and methods}

\subsection{Cases and healthy controls}

Throughout the period between April 2007 and April 2010, we identified RBD cases attending 13 European treatment centers from 11 countries: Belgium (2), Denmark, Germany (2), Greece, France, Ireland, Italy, Serbia, Slovenia, Turkey, and the United Kingdom. Data on these patients were collected as part of the EN-RBD project which is described elsewhere [2]. The project was approved by the Ethical Review Board of the Fondazione IRCCS Ca' Granda Ospedale Maggiore Policlinico, University of Milan, Italy, in compliance with all pertinent national and international ethical standards. Written informed consent was obtained from each participating patient.

We included in this study 492 patients with a known bleeding history who were already diagnosed by the participating centers after presenting with bleeding episodes, through preoperative screening, or through family screening. The diagnosis of a coagulation deficiency was based on the measurement of the residual factor plasma activity level below the normal thresholds [2].
We also recruited 107 healthy controls during the same time period. Healthy controls were recruited from among the staff of Milan hospital, their friends or neighbors, they were Italian, unrelated to patients and eligible if in good health and if never referred for hemostasis evaluation.

\subsection{The bleeding questionnaire and score system}

A questionnaire on bleeding symptoms (Table 1) was administered to each enrolled patient by the referring physician. The bleeding questionnaire was administered to healthy controls by the same doctor who administered the questionnaire to the Italian patients.

For this study, we retrieved data on demographics (age at data collection and sex) and bleeding history up to the date of patient enrollment, including the type (site) of bleeding and its characteristics: frequency, spontaneity, extent, localization, whether it occurred while the patient was on prophylaxis, and type of treatment used to control the bleeding.

For each patient and healthy control subject, retrieved data on bleeding history were categorized according to the type of bleeding and its characteristics, with each item given a score of 1 for presence and 0 for absence as described in Table 1 . A score of 1 was given if the subject had experienced the type of bleeding and additionally for each characteristic representing a more severe type of bleeding (frequent, spontaneous, exposure/occurrence while on prophylaxis, extensive, required treatment). When more than one option of treatment was available, higher scores ( 2 or 3 ) were given for more aggressive modalities of therapy. Scoring for menorrhagia and for postpartum bleeding was only done for women. Scoring for postpartum bleeding, tooth extraction, tonsillectomy, minor or major surgery was only done for subjects who underwent the procedures. Subjects who underwent these procedures but did not have a bleeding outcome were given the score of -1 ; in case of prophylaxis administered before the surgery a score of 1 was given, so that the total score for that procedure was 0 . These scores were assigned based on consensus agreement between the study investigators, and guided by scoring tools used for other bleeding disorders [17]. For each type of bleeding, an 'index score' was calculated from the sum of scores assigned for all items within its category.

An analysis was conducted to compare our BSS with the ISTH-BAT [10] whose publication coincided with the end of our data collection. The comparison was done on the subgroup of Italian patients/controls whose more specific information requested in the ISTH-BAT questionnaire were easily retrieved from medical records or by interviewing patients/controls. To evaluate whether our BSS can identify the severity rather than only the diagnosis of RBD, we conducted further analysis on patients with RBD to evaluate the correlation and predictive power for our BS against the coagulant factor activity level.

\subsection{Statistical analysis}

Data are presented as means, ranges or percentages. The BS was extracted from the Z-value of a multivariate logistic regression model, in which the outcome RBD versus no RBD was the dependent variable and the symptoms (risk factors) were the independent variables. Covariates entered in the model were age, sex, and the index scores for each type of bleeding. Whether the BS was associated with a diagnosis of RBD was tested by a receiver-operator curve (ROC) analysis. The probability of having a RBD can be calculated from the bleeding score using the formula:

Probability of RBD $=1 / 1+\mathrm{e}-[\mathrm{BS}]$

To compare the performance of our BSS against the ISTH-BAT, we evaluated the correlation between the two score on Pearson's correlation analysis and compared the areas under the curve (AUC) and their $95 \%$ confidence intervals $(\mathrm{CI})$ on a ROC curve analysis for the diagnosis of RBD. 
Table 1

Lifelong clinical bleeding scoring system.

\begin{tabular}{|c|c|c|}
\hline Parameter & Key & Value \\
\hline Epistaxis & No $=0$, Yes $=1$ & \\
\hline Frequency $>1 /$ year & No $=0$, Yes $=1$ & \\
\hline Spontaneous & No $=0$, Yes $=1$ & \\
\hline On prophylaxis & No $=0$, Yes $=1$ & \\
\hline Diffuse bleeding ${ }^{\mathrm{a}}$ & No $=0$, Yes $=1$ & \\
\hline Bilateral & No $=0$, Yes $=1$ & \\
\hline $\begin{array}{l}\text { Requiring treatment with packing or } \\
\text { cauterization or antifibrinolytics }\end{array}$ & No $=0$, Yes $=1$ & \\
\hline $\begin{array}{l}\text { Requiring treatment with blood } \\
\text { transfusion or replacement therapy or } \\
\text { desmopressin }\end{array}$ & No $=0$, Yes $=2$ & \\
\hline Epistaxis index score (Epi_In) & Total sum & \\
\hline Oral cavity bleeding ${ }^{\mathrm{b}}$ & No $=0$, Yes $=1$ & \\
\hline Frequency > 1/year & No $=0$, Yes $=1$ & \\
\hline Spontaneous & No $=0$, Yes $=1$ & \\
\hline On prophylaxis & No $=0$, Yes $=1$ & \\
\hline Diffuse bleeding $^{\mathrm{a}}$ & No $=0$, Yes $=1$ & \\
\hline Involving tongue & No $=0$, Yes $=1$ & \\
\hline $\begin{array}{l}\text { Requiring treatment with surgical } \\
\text { hemostasis or antifibrinolytics }\end{array}$ & No $=0$, Yes $=1$ & \\
\hline $\begin{array}{l}\text { Requiring treatment with blood } \\
\text { transfusion or replacement therapy or } \\
\text { desmopressin }\end{array}$ & No $=0$, Yes $=2$ & \\
\hline Oral cavity bleeding index score (Oral_In) & Total sum & \\
\hline Bruising & No $=0$, Yes $=1$ & \\
\hline Frequency $\geq 1 /$ year & No $=0$, Yes $=1$ & \\
\hline Spontaneous & No $=0$, Yes $=1$ & \\
\hline On prophylaxis & No $=0$, Yes $=1$ & \\
\hline Size $>5 \mathrm{~cm}$ & No $=0$, Yes $=1$ & \\
\hline Involving head or thorax & No $=0$, Yes $=1$ & \\
\hline Requiring consultation & No $=0$, Yes $=1$ & \\
\hline Bruising index score (Bruis_In) & Total sum & \\
\hline Hematoma & No $=0$, Yes $=1$ & \\
\hline Frequency $>1$ & No $=0$, Yes $=1$ & \\
\hline Spontaneous & No $=0$, Yes $=1$ & \\
\hline On prophylaxis & No $=0$, Yes $=1$ & \\
\hline Diffuse bleeding $^{\mathrm{a}}$ & No $=0$, Yes $=1$ & \\
\hline $\begin{array}{l}\text { Requiring treatment with replacement } \\
\text { therapy or desmopressin }\end{array}$ & No $=0$, Yes $=1$ & \\
\hline $\begin{array}{l}\text { Requiring treatment with } \\
\text { surgery }+ \text { blood transfusion or } \\
\text { replacement therapy }\end{array}$ & No $=0$, Yes $=2$ & \\
\hline Hematoma index score (Hemato_In) & Total sum & \\
\hline Hemarthrosis & No $=0$, Yes $=1$ & \\
\hline Frequency $>1$ & No $=0$, Yes $=1$ & \\
\hline Spontaneous & No $=0$, Yes $=1$ & \\
\hline On prophylaxis & No $=0$, Yes $=1$ & \\
\hline Disabling $^{\mathrm{C}}$ & No $=0$, Yes $=1$ & \\
\hline $\begin{array}{l}\text { Requiring treatment with replacement } \\
\text { therapy or desmopressin }\end{array}$ & No $=0$, Yes $=1$ & \\
\hline $\begin{array}{l}\text { Requiring treatment with blood } \\
\text { transfusion }\end{array}$ & No $=0$, Yes $=2$ & \\
\hline Hemarthrosis index score (Hemar_In) & Total sum & \\
\hline Gastrointestinal bleeding & No $=0$, Yes $=1$ & \\
\hline Frequency $\geq 2$ times & No $=0$, Yes $=1$ & \\
\hline Spontaneous ${ }^{\mathrm{d}}$ & No $=0$, Yes $=1$ & \\
\hline On prophylaxis & No $=0$, Yes $=1$ & \\
\hline Causing anemia & No $=0$, Yes $=1$ & \\
\hline Requiring treatment with antifibrinolytics & No $=0$, Yes $=1$ & \\
\hline $\begin{array}{l}\text { Requiring treatment with surgical } \\
\text { hemostasis, blood transfusion, } \\
\text { replacement therapy or desmopressin }\end{array}$ & No $=0$, Yes $=2$ & \\
\hline Gastrointestinal bleeding index score (GI_In) & Total sum & \\
\hline CNS bleeding & No $=0$, Yes $=1$ & \\
\hline Spontaneous & No $=0$, Yes $=1$ & \\
\hline On prophylaxis & No $=0$, Yes $=1$ & \\
\hline Diffuse bleeding ${ }^{\mathrm{a}}$ & No $=0$, Yes $=1$ & \\
\hline Intracerebral bleeding & No $=0$, Yes $=1$ & \\
\hline $\begin{array}{l}\text { Requiring treatment with replacement } \\
\text { therapy }\end{array}$ & No $=0$, Yes $=1$ & \\
\hline $\begin{array}{l}\text { Requiring treatment with } \\
\text { surgery }+ \text { blood transfusion or } \\
\text { replacement therapy }\end{array}$ & No $=0$, Yes $=1$ & \\
\hline CNS bleeding index score (CNS_In) & Total sum & \\
\hline
\end{tabular}

Table 1 (continued)

\begin{tabular}{|c|c|c|}
\hline Parameter & Key & Value \\
\hline Menorrhagia & No or Male $=0$, Yes $=1$ & \\
\hline Frequency $\geq 1 /$ year & No or Male $=0$, Yes $=1$ & \\
\hline Spontaneous & No or Male $=0$, Yes $=1$ & \\
\hline On prophylaxis & No or Male $=0$, Yes $=1$ & \\
\hline Pictorial chart $\geq 185$ & No or Male $=0$, Yes $=1$ & \\
\hline $\begin{array}{l}\text { Requiring treatment with antifibrinolytics, } \\
\text { pill use, iron therapy or curettage }\end{array}$ & No or Male $=0$, Yes $=1$ & \\
\hline $\begin{array}{l}\text { Requiring treatment with blood } \\
\text { transfusion or replacement therapy or } \\
\text { desmopressin }\end{array}$ & No or Male $=0$, Yes $=2$ & \\
\hline Requiring treatment with hysterectomy & No or Male $=0$, Yes $=3$ & \\
\hline Menorrhagia index score (Meno_In) & Total sum & \\
\hline Postpartum bleeding & $\begin{array}{l}\text { Never delivered or } \\
\text { Male }=0 \text {, No }=-1 \\
\text { Yes }=1\end{array}$ & \\
\hline Frequency $>1$ time & $\begin{array}{l}\text { Never delivered or } \\
\text { Male }=0, \text { No }=0 \\
\text { Yes }=1\end{array}$ & \\
\hline Spontaneous ${ }^{\mathrm{e}}$ & $\begin{array}{l}\text { Never delivered or } \\
\text { Male }=0 \text {, No }=0 \text {, } \\
\text { Yes }=1\end{array}$ & \\
\hline On prophylaxis & $\begin{array}{l}\text { Never delivered or } \\
\text { Male }=0 \text {, No }=0 \text {, } \\
\text { Yes }=1\end{array}$ & \\
\hline Causing anemia & $\begin{array}{l}\text { Never delivered or } \\
\text { Male }=0 \text {, No }=0 \text {, } \\
\text { Yes }=1\end{array}$ & \\
\hline $\begin{array}{l}\text { Requiring treatment with antifibrinolytics, } \\
\text { iron therapy or curettage }\end{array}$ & $\begin{array}{l}\text { Never delivered or } \\
\text { Male }=0 \text {, No }=0 \text {, } \\
\text { Yes }=1\end{array}$ & \\
\hline $\begin{array}{l}\text { Requiring treatment with blood } \\
\text { transfusion or replacement therapy or } \\
\text { desmopressin }\end{array}$ & $\begin{array}{l}\text { Never delivered or } \\
\text { Male }=0, \text { No }=0 \\
\text { Yes }=2\end{array}$ & \\
\hline Requiring treatment with hysterectomy & $\begin{array}{l}\text { Never delivered or } \\
\text { Male }=0 \text {, No }=0 \text {, } \\
\text { Yes }=3\end{array}$ & \\
\hline Postpartum bleeding index score (PPH_In) & Total sum & \\
\hline Bleeding during tooth extraction & $\begin{array}{l}\text { Never done }=0 \\
\text { No }=-1, \text { Yes }=1\end{array}$ & \\
\hline Frequency $>1$ time & $\begin{array}{l}\text { Never done }=0 \text {, No }=0 \text {, } \\
\text { Yes }=1\end{array}$ & \\
\hline On prophylaxis & $\begin{array}{l}\text { Never done }=0 \text {, No }=0 \\
\text { Yes }=1\end{array}$ & \\
\hline For $\geq 15 \mathrm{~min}$ & $\begin{array}{l}\text { Never done }=0 \text {, No }=0 \\
\text { Yes }=1\end{array}$ & \\
\hline Recurrence of bleeding & $\begin{array}{l}\text { Never done }=0 \text {, No }=0 \\
\text { Yes }=1\end{array}$ & \\
\hline $\begin{array}{l}\text { Requiring treatment with resuturing or } \\
\text { packing or antifibrinolytics }\end{array}$ & $\begin{array}{l}\text { Never done }=0 \text {, No }=0 \text {, } \\
\text { Yes }=1\end{array}$ & \\
\hline $\begin{array}{l}\text { Requiring treatment with blood } \\
\text { transfusion or replacement therapy or } \\
\text { desmopressin }\end{array}$ & $\begin{array}{l}\text { Never done }=0 \text {, No }=0 \\
\text { Yes }=2\end{array}$ & \\
\hline $\begin{array}{l}\text { Tooth extraction bleeding index score } \\
\text { (Tooth_In) }\end{array}$ & Total sum & \\
\hline Bleeding during minor surgery ${ }^{\mathrm{f}}$ & $\begin{array}{l}\text { Never done }=0 \\
\text { No }=-1 \text {, Yes }=1\end{array}$ & \\
\hline Frequency $>1$ time & $\begin{array}{l}\text { Never done }=0 \text {, No }=0 \\
\text { Yes }=1\end{array}$ & \\
\hline On prophylaxis & $\begin{array}{l}\text { Never done }=0 \text {, No }=0 \\
\text { Yes }=1\end{array}$ & \\
\hline Postoperative bleeding & $\begin{array}{l}\text { Never done }=0 \text {, No }=0 \\
\text { Yes }=1\end{array}$ & \\
\hline $\begin{array}{l}\text { Requiring treatment with surgical } \\
\text { hemostasis or antifibrinolytics }\end{array}$ & $\begin{array}{l}\text { Never done }=0 \text {, No }=0 \\
\text { Yes }=1\end{array}$ & \\
\hline $\begin{array}{l}\text { Requiring treatment with blood } \\
\text { transfusion or replacement therapy or } \\
\text { desmopressin }\end{array}$ & $\begin{array}{l}\text { Never done }=0 \text {, No }=0 \\
\text { Yes }=2\end{array}$ & \\
\hline $\begin{array}{l}\text { Minor surgery bleeding index score } \\
\text { (Minor_In) }\end{array}$ & Total sum & \\
\hline Bleeding during tonsillectomy ${ }^{\mathrm{g}}$ & $\begin{array}{l}\text { Never done }=0 \\
\text { No }=-1, \text { Yes }=1\end{array}$ & \\
\hline On prophylaxis & $\begin{array}{l}\text { Never done }=0 \text {, No }=0 \text {, } \\
\text { Yes }=1\end{array}$ & \\
\hline Postoperative bleeding & $\begin{array}{l}\text { Never done }=0 \text {, No }=0 \\
\text { Yes }=1\end{array}$ & \\
\hline
\end{tabular}


Table 1 (continued)

\begin{tabular}{lll}
\hline Parameter & Key & Value \\
\hline $\begin{array}{l}\text { Requiring treatment with surgical } \\
\text { hemostasis or antifibrinolytics }\end{array}$ & Never done $=0$, No $=0$, \\
Requiring treatment with blood & Yes $=1$ \\
transfusion or replacement therapy or & Never done $=0$, No $=0$, \\
desmopressin & Yes $=1$ \\
Tonsillectomy bleeding index score & \\
(Tonsil_In) & Total sum \\
Bleeding during major surgery ${ }^{\mathrm{f}}$ & Never done $=0$, \\
& No $=-1$, Yes $=1$ \\
Frequency $>1$ time & Never done $=0$, No $=0$, \\
& Yes $=1$ \\
On prophylaxis & Never done $=0$, No $=0$, \\
& Yes $=1$ \\
Postoperative bleeding & Never done $=0$, No $=0$, \\
& Yes $=1$ \\
Requiring treatment with surgical & Never done $=0$, No $=0$, \\
hemostasis or antifibrinolytics & Yes $=1$ \\
Requiring treatment with blood & Never done $=0$, No $=0$, \\
transfusion or replacement therapy or & Yes $=2$ \\
desmopressin & \\
Major surgery bleeding index score & Total sum \\
(Major_In) & \\
\hline
\end{tabular}

CNS, central nervous system.

a Diffuse bleeding: bleeding lasting at least $10 \mathrm{~min}$ and/or requiring medical attention in case of epistaxis or oral cavity bleeding; hematoma larger than $3 \mathrm{~cm}$ or considered disproportionate to trauma by the investigator in case of hematoma, when it is not possible to establish a localized lesion and define the hemorrhage volume in case of CNS bleeding.

b Oral cavity bleeding includes gum bleeding, tooth eruption or spontaneous tooth loss bleeding, bleeding occurring after bites/wounds to lips, cheek and tongue. The gum bleeding due to toothbrush should be evaluated as normal; gum bleeding should be considered significant when it causes frankly bloody sputum.

c Disabling means that hemarthrosis has led to a compromised motility.

d Any gastrointestinal bleeding that is not explained by the presence of a specific disease should be considered as spontaneous. Bleeding associated with ulcer, portal hypertension, hemorrhoids or angiodysplasia should be considered as not spontaneous. Melena and hematemesis, if not associated with the conditions mentioned above, should be considered possible signs of bleeding disorders.

e Postpartum bleeding should be considered as spontaneous when it is not associated with an identified gynecological or obstetrical cause, as for example the commonest causes of primary PPH (uterine atony, cervical laceration, ....).

${ }^{\mathrm{f}}$ Definition of minor and major surgery are described in [16].

$\mathrm{g}$ With or without adenoidectomy.

To evaluate the correlation between our BS and coagulant factor activity level, Pearson's correlation analysis was performed. A ROC curve analysis was also conducted to establish the predictive power of our BS in identifying severe compared with mild-moderate RBD, as defined previously based on coagulant factor activity level [17].

Analysis was carried out using SPSS v.19 (SPSS Inc., Chicago, IL, USA)

Table 2

Proportions of patients and controls with different types of bleeding

\begin{tabular}{|c|c|c|c|}
\hline Type of bleeding & $\begin{array}{l}\text { RBD patients } \\
\mathrm{n} / \mathrm{N}(\%, 95 \% \mathrm{CI})\end{array}$ & $\begin{array}{l}\text { Controls } \\
\mathrm{n} / \mathrm{N}(\%, 95 \% \mathrm{CI})\end{array}$ & p-Value \\
\hline Epistaxis & $133 / 492(27.0,23.3-31.1)$ & $40 / 107(37.4,28.8-46.9)$ & 0.032 \\
\hline Oral cavity bleeding & $81 / 492(16.5,13.5-20.0)$ & $12 / 107(11.2,6.6-18.6)$ & 0.174 \\
\hline Bruising & $133 / 492(27.0,23.3-31.1)$ & $51 / 107(47.7,38.4-57.1)$ & $<0.001$ \\
\hline Hematoma & $85 / 492(17.3,14.2-20.9)$ & $24 / 107(22.4,15.6-31.3)$ & 0.210 \\
\hline Hemarthrosis & $40 / 492(8.1,6.0-10.9)$ & $7 / 107(6.5,3.3-12.9)$ & 0.580 \\
\hline Gastrointestinal bleeding & $37 / 492(7.5,5.5-10.2)$ & $2 / 107(1.9,0.5-6.5)$ & 0.032 \\
\hline CNS bleeding & $35 / 492(7.1,5.2-9.7)$ & $1 / 107(0.9,0.2-5.1)$ & 0.015 \\
\hline Menorrhagia $^{a}$ & $70 / 204(34.3,28.1-41.1)$ & $10 / 47(21.3,12.0-35.0)$ & 0.084 \\
\hline Postpartum bleeding ${ }^{\mathrm{a}, \mathrm{b}}$ & $22 / 109(20.2,13.7-28.7)$ & $3 / 18(16.7,6.1-39.6)$ & 0.728 \\
\hline Bleeding during tooth extraction ${ }^{\mathrm{b}}$ & $67 / 168(39.9,32.8-47.4)$ & $8 / 72(11.1,5.8-20.5)$ & $<0.001$ \\
\hline Bleeding during minor surgery ${ }^{\mathrm{b}}$ & $19 / 100(19.0,12.5-27.8)$ & $1 / 59(1.7,0.4-8.9)$ & 0.001 \\
\hline Bleeding during tonsillectomy ${ }^{\mathrm{b}}$ & $23 / 66(34.8,24.5-46.9)$ & $2 / 23(8.7,2.7-27.0)$ & 0.016 \\
\hline Bleeding during major surgery ${ }^{\mathrm{b}}$ & $32 / 148(21.6,15.8-28.9)$ & $5 / 50(10.0,4.4-21.4)$ & 0.068 \\
\hline
\end{tabular}

RBD, rare bleeding disorders; CNS, central nervous system.

a Only women included.

b Only patients undergoing the procedure were included.

\section{Results}

Data from 492 RBD patients and 107 healthy controls were analyzed. RBD patients most commonly had FVII $(\mathrm{n}=203,41.3 \%)$ and FXI $(\mathrm{n}=$ $117,23.8 \%$ ) deficiencies followed by fibrinogen $(n=43,8.7 \%), F V$ ( $n=43,8.7 \%)$, FXIII $(n=33,6.7 \%), F X(n=31,6.3 \%)$, combined FV and FVIII $(\mathrm{n}=16,3.3 \%)$, and FII $(\mathrm{n}=6,1.2 \%)$ deficiencies with all laboratory severities. The mean age of patients was lower than controls (29.3 \pm 18.0 [range $1-95$ ] versus 35.2 years \pm 15.3 [range $3-72$ ], mean difference: $5.8,95 \% \mathrm{CI}: 2.4-9.3)$, while the sex distribution was comparable (47.6\% [95\% CI: 43.2-52.0] versus 51.4\% [95\% CI: $42.0-$ 60.7] men). The proportions of patients and controls that had different types of bleeding are summarized in Table 2. A history of most types of bleeding was generally more common in RBD patients than controls, except for epistaxis, bruising, or hematoma. come of RBD versus no RBD are summarized in Table 3. On the basis of these results, the $Z$-value representing the bleeding score can be calculated using the formula in which the sum of beta of each risk factor times the risk factor index plus constant are taken into account:

Bleeding Score $(\mathrm{BS})=2.510+($ Age in years $\times-0.029)+(-0.305$ if Male $)+($ Epi_In $\times-0.129)+($ Oral_In $\times 0.197)+($ Bruis_In $\times-$ $0.342)+($ Hemato_In $\times-0.040)+($ Hemar_In $\times 0.618)+$ $($ GI_In $\times$ 0.490 $)+($ CNS_In $\times 0.876)+($ Meno_In $\times$ 0.073 $)+$ $($ PPH_In $\times 0.334)+($ Tooth_In $\times 0.277)+($ Minor_In $\times 0.270)+$ $($ Tonsil_In $\times 0.670)+($ Major_In $\times 0.281)$.

As shown in Table 3, the weight of the diverse symptoms (risk factors) in determining the bleeding score is different with that of CNS bleeding, bleeding during tonsillectomy and hemarthrosis being highest.

According to the BS formula, the mean BS was 2.0 (range, -0.6-13.6) and was higher in patients than controls ( 2.1 versus 1.2 , mean difference: $0.9,95 \% \mathrm{CI}$ : 0.8-1.1). On ROC curve analysis, the bleeding score value of 1.5 had the highest sum of sensitivity (67.1\%) and specificity (73.8\%) in discriminating patients with RBD from those without (area under the curve: 0.73, 95\% CI: 0.69-0.78).

The probability of having a RBD can be calculated from the bleeding score using the following formula:

Probability of $\mathrm{RBD}=1 /\left(1+\mathrm{e}^{-[\mathrm{BS}]}\right)$

Supplement 1 is a calculator to facilitate calculation of both the bleeding score and the probability of having RBD given patients age, sex, and bleeding history. Note that to normalize baseline probability, which may be specific to the types of controls in this study, to $50 \%$, the
Results from the multivariate logistic regression model for the out- 
Table 3

Multivariate logistic regression model showing effect estimates for the outcome of rare bleeding disorder (Yes versus No).

\begin{tabular}{|c|c|c|c|c|c|c|c|c|}
\hline \multirow[t]{2}{*}{ Variable } & \multirow[t]{2}{*}{ Beta } & \multirow[t]{2}{*}{ S.E. } & \multirow[t]{2}{*}{ Wald } & \multirow[t]{2}{*}{ df } & \multirow[t]{2}{*}{$p$-Value } & \multirow[t]{2}{*}{ OR } & \multicolumn{2}{|l|}{$95 \% \mathrm{CI}$} \\
\hline & & & & & & & Lower & Upper \\
\hline Age & -0.029 & 0.006 & 20.575 & 1 & 0.000 & 0.971 & 0.959 & 0.983 \\
\hline Sex (male versus female) & -0.305 & 0.243 & 1.573 & 1 & 0.210 & 0.737 & 0.457 & 1.188 \\
\hline Epistaxis index score (Epi_In) & -0.129 & 0.074 & 3.021 & 1 & 0.082 & 0.879 & 0.760 & 1.017 \\
\hline Oral cavity bleeding index score (Oral_In) & 0.197 & 0.144 & 1.859 & 1 & 0.173 & 1.218 & 0.918 & 1.616 \\
\hline Bruising index score (Bruis_In) & -0.342 & 0.113 & 9.230 & 1 & 0.002 & 0.710 & 0.570 & 0.886 \\
\hline Hematoma index score (Hemato_In) & -0.040 & 0.138 & 0.085 & 1 & 0.770 & 0.960 & 0.732 & 1.260 \\
\hline Hemarthrosis index score (Hemar_In) & 0.618 & 0.262 & 5.561 & 1 & 0.018 & 1.855 & 1.110 & 3.101 \\
\hline Gastrointestinal bleeding index score (GI_In) & 0.490 & 0.298 & 2.692 & 1 & 0.101 & 1.632 & 0.909 & 2.928 \\
\hline CNS bleeding index score (CNS_In) & 0.876 & 0.546 & 2.569 & 1 & 0.109 & 2.400 & 0.823 & 7.004 \\
\hline Menorrhagia index score (Meno_In) & 0.073 & 0.103 & 0.504 & 1 & 0.478 & 1.076 & 0.879 & 1.317 \\
\hline Postpartum bleeding index score (PPH_In) & 0.334 & 0.236 & 2.017 & 1 & 0.156 & 1.397 & 0.881 & 2.217 \\
\hline Tooth extraction bleeding index score (Tooth_In) & 0.277 & 0.143 & 3.746 & 1 & 0.053 & 1.319 & 0.996 & 1.747 \\
\hline Minor surgery bleeding index score (Minor_In) & 0.270 & 0.327 & 0.680 & 1 & 0.409 & 1.310 & 0.690 & 2.486 \\
\hline Tonsillectomy bleeding index score (Tonsil_In) & 0.670 & 0.390 & 2.954 & 1 & 0.086 & 1.954 & 0.910 & 4.193 \\
\hline Major surgery bleeding index score (Major_In) & 0.281 & 0.191 & 2.173 & 1 & 0.140 & 1.325 & 0.911 & 1.926 \\
\hline Constant & 2.510 & 0.291 & 74.318 & 1 & 0.000 & 12.300 & - & - \\
\hline
\end{tabular}

CNS, central nervous system; S.E., standard error; df, degrees of freedom; OR, odds ratio; CI, confidence interval.

constant (2.51) is removed from the BS before inclusion into the probability formula.

We conducted separate analysis on the Italian RBD patients $(\mathrm{n}=$ 141 , mean age $39.4 \pm 20.0$ years, $60.3 \%$ women $)$ and controls $(\mathrm{n}=$ 107 , mean age $35.2 \pm 15.4$ years, $48.6 \%$ ), to evaluate the predictive power of our BS model compared to the ISTH-BAT score. Similar to the data form the total population, the BS was higher in Italian patients than in controls in our model $(2.2 \pm 1.8$ vs $1.2 \pm 0.6, p<0.001)$. The ISTH-BAT score was also higher in Italian patients than in controls $(4.9 \pm 5.5$ vs $1.7 \pm 2.2, p<0.001)$, although with higher dispersion. There was statistically significant correlation between the two scores (Pearson's correlation $r=0.681, p<0.001$ ). On ROC curve analysis for the diagnosis of an RBD, the AUC for the two scores were comparable indicating similar predictive power (BS score in this study: AUC 0.709, 95\% CI: $0.645-0.773, p<0.001$ vs ISTH-BAT: AUC: $0.690,95 \%$ CI: $0.624-$ $0.755, p<0.001$ ).

In RBDs patients, there was a significant negative correlation between BS and coagulant factor activity level (Pearson's correlation $r=-0.215, p<0.001$ ); which was strongest for fibrinogen (Pearson's correlation $\mathrm{r}=-0.538, p<0.001$ ) and FXIII (Pearson's correlation $\mathrm{r}=-0.525, p=0.002$ ) deficiencies. A BS of $>3.2$ was the strongest predictor (highest sum of sensitivity and specificity) of severe compared with mild-moderate fibrinogen deficiency (AUC: 0.935, 95\% CI: $0.856-$ $0.999, p<0.001$ ) while a level of $>3.7$ was the strongest predictor of severe compared with mild-moderate FXIII deficiency (AUC: $0.857,95 \%$ CI: $0.718-0.996, p=0.001)$.

\section{Discussion}

A detailed bleeding history is an important component of the medical evaluation to improve diagnostic accuracy, thus avoid unwarranted laboratory testing, to describe symptom severity and to inform treatment.

Until now different scoring systems have been developed. Unfortunately, the majority of them were designed for patients with bleeding disorders other than RBDs. Moreover, currently available tools have some limitations; some of them lack pediatric-specific symptoms while others accentuate the severity of bleeding symptoms over other potentially clinically important features, such as the frequency of symptoms. Finally, the existing scoring systems were designed based on the interpretation of the bleeding symptoms with a priori criteria. Such "external" system has the advantage that it is not data driven and no validation is required, but it has the disadvantage that it is completely arbitrary and scales could be different for men and women. At variance, a data driven scoring system ("internal" system), starting from the interpretation of symptoms of already diagnosed patients, is optimistic, but objective because it scores each symptom and there are no different scales for different groups of people.

In our study, we developed a formula to calculate the probability of having a RBD based on a BSS constructed by retrieving data related to patients with RBDs. The BSS presented herein was able to differentiate patients with RBDs from normal controls; and was able to distinguish RBD patients that could potentially have severe deficiency levels for fibrinogen and FXIII. This BSS was designed to circumvent the wellknown issues related to age and sex and the time of the administration of the bleeding questionnaire. An increase of the BS as a function of age has been reported in individuals with bleeding disorders $[7,8,18]$. In healthy individuals, however, some studies reported no correlation between age and number of bleeding symptoms $[7,8,18]$, while others reported some degree of association and even a modest decrease in the reported frequency of epistaxis with age, suggesting either that epistaxis is becoming more common or that older individuals are less likely to remember epistaxis from their youth [4]. Sex-related issues should also be considered since women, in addition to usual bleeding symptoms, are likely to experience bleedings associated to obstetrical and gynecological problems, pregnancy and childbirth. Although differences in the number of reported bleeding symptoms did not persist after exclusion of sex-specific questions $[7,8]$, some symptoms such as easy bruising and bruising after venipuncture were shown to be more common in women than in men $[4,19]$. With these considerations in mind, in our multivariate logistic regression model we added age and sex as covariates, thus taking into account their predictive effect on the probability of having a RBD.

With regard to using BATs as a screening tool for bleeding disorders, it is important to recognize how specific study populations can affect the results. This is important if symptoms from individuals known to have a bleeding disorder are included after diagnosis, when prophylactic treatments might have been given. The previous VWD-BATs publications dealt with this issue in different ways, by studying obligatory carriers eliminating the possibility of increasing the sensitivity by studying known bleeders [8], or by evaluating only bleeding symptoms present before the diagnosis or symptoms from individuals who did not receive hemostatic prophylaxis [20]. Since the EN-RBD database captured data through retrospective review of medical records, it lacks detailed data on the diagnosis setting and the exact chronology between diagnosis and bleeding episodes in some instances. Therefore, in order to try to eliminate this potential source of bias, we chose to include a question related to whether the bleeding occurred while the patient was on prophylaxis. This question was considered in each bleeding index score and thus was part of the covariates used to build up the 
formula to calculate the risk of a RBD. Finally, our bleeding questionnaire, although including questions related to the most common symptoms reported in RBDs patients, is relatively short and easy to administer. This BS correlated well with the ISTH-BAT, and had a comparable predictive power for the diagnosis of RBD. The added advantage for our BS over that of the ISTH-BAT is that it was specifically developed using data from RBD patients and the questions were tailored for this subgroup of bleeding disorders; thus, could carry independent clinical value when the diagnosis of RBD in specific is suspected. Moreover, since the AUC and sensitivity and specificity for both scores do not extend to $>0.75$ and $>90 \%$, respectively, leaving room for false positive and negative predictions, the 'probability of RBD' calculation provided as adjunct to the BS could aid in the diagnosis by the concerned health care provider especially when combined with other clinical and diagnostic clues. Nonetheless, we believe that further studies are required to identify the most discriminatory questions and to potentially create a shorter questionnaire that focuses on data with a better diagnostic efficiency.

Our study has some limitations. First, all the healthy controls were Italian; therefore, our tool may have different profiles if controls from another country were enrolled. Ideally, we should have enrolled controls from the same countries as patients. On the other hand, the literature on differences in bleeding symptoms as a function of race and ethnicity is sparse. The only observation that addressed this issue is related to a lower frequency of epistaxis among Asians [21], or AfricanAmericans than among Caucasians [4]. Nevertheless, our patients group is only composed of Caucasians, as the Italian controls are. Second, the physician undergoing the assessment was not blinded to whether the subject was a patient or control, which may have influenced the depth of interview to retrieve required information for the scoring questionnaire. Another limitation comes from the data-driven nature of the BSS. This is a score based on data retrieved from the ENRBD database, therefore its validation is needed in another setting of individuals to verify its positive and negative predictive values. The validation is also warranted to assess its clinical utility in patients with bleeding symptoms severe enough to undergo an investigation of a bleeding disorder, such as hemophilia and VWD. Future collaborative studies are therefore needed to test the validity and reliability of the present BSS. It should also be noted that RBDs, each here represented by a different number of patients, were demonstrated to be a heterogeneous group of bleeding disorders [2,17], characterized by different frequencies of types of bleeding symptoms. Therefore, specific studies, probably including the BSS, should be focused on each individual RBD independently. Only a population-based screening effort could circumvent this, which, however, is unfeasible given the rarity of the disorders.

\section{Conclusions}

Using data retrieved from a large group of patients with RBDs and healthy individuals, we provided a new BSS that could help distinguish patients with RBDs from normal controls based on their demographics and bleeding history. We also created an easy-to-use formula that generates the probability of having a RBD. The use of such a tool during the initial patient interview may represent a valuable support tool to clinicians in identifying patients who really need subsequent diagnostic workup. A similar approach to BSS construction is ongoing with the aim of predicting the incidence and severity of future bleeding episodes in patients with RBDs utilizing a BSS retrieved at a baseline encounter.

Supplementary data to this article can be found online at http://dx doi.org/10.1016/j.thromres.2016.11.008.

\section{Funding}

This work is part of the project "Establishment of a European Network of Rare Bleeding Disorders (EN-RBD)" funded by the European Union in the framework of the Public Health Programme (agreement number 2006118). The funder had no involvement in study design, data collection, data analysis, manuscript preparation and publication decision.

\section{Author contribution}

R. Palla designed the study, analyzed data and wrote the paper; S. M. Siboni and M. Menegatti were involved in the design of the study and collected information on patients affected with RBDs and on healthy controls; K. M. Musallam performed statistical analyses and assisted in drafting the manuscript; F. Peyvandi supervised the study and critically revised the paper. All authors approved the final version of the manuscript before submission.

\section{Diclosures}

R. Palla and M. Menegatti have received travel support from Pfizer. F. Peyvandi has received honoraria for participating as a speaker at satellite symposia and educational meetings organized by Bayer, Biotest, CSL Behring, Grifols, Novo Nordisk, and Sobi. She is recipient of research grant funding from Alexion, Biotest, Kedrion Biopharma, and Novo Nordisk paid to Fondazione Luigi Villa, and she has received consulting fees from Kedrion Biopharma, LFB and Octapharma. She is member of the Ablynx scientific advisory board. These activities fall outside the present work. Remaining authors stated that they had no interests which might be perceived as posing a conflict or bias.

\section{Appendix A. Appendix}

List of the collaborating partners involved in the EN-RBD project (www.rbdd.eu/partners.htm):

- Dr. Majda Benedik-Dolnicar and Dr. Lidija Kitanovski (National Haemophilia Center, University Children's Hospital, Ljubljiana, Slovenia),

- Dr. Christoph Bidlingmaier (Pediatric Hemophilia and Thrombosis Centre, Dr. von Hauner's Children's University Hospital, Munich, Germany),

- Prof. Tiraje Celkan and Dr. Nihal Özdemir (Department of Pediatric Hematology-Oncology, Cerrahpasa Medical Faculty of Istanbul University, Istanbul, Turkey),

- Prof. Alain Gadisseur (Antwerp University Hospital UZA, Edegem, Belgium),

- Dr. Paul Giangrande (Oxford Haemophilia \& Thrombosis Centre, Nuffield Department of Clinical Medicine, University of Oxford, Oxford, United Kingdom),

- Dr. Ruth Gilmore (National Centre for Hereditary Coagulation Disorders, St James's Hospital, Dublin, Ireland),

- Dr. Susan Halimeh (Coagulation center Rhine Ruhr (GZRR), Duisburg, Germany),

- Prof. Jorgen Ingerslev (Centre for Hemophilia and Thrombosis, Dept. of Clinical Biochemistry, University Hospital Skejbi, Aarhus, Denmark),

- Dr. Danijela Mikovic (Haemostasis Department and Haemophilia Center, Blood Transfusion Institute of Serbia, Belgrade, Serbia),

- Dr. Kathelijene Peerlink (Hemofilie Centrum Leuven, Katholieke Universiteit, Leuven, Belgium),

- Prof. Helen Platokouki, Dr. Helen Pergantou (Haemophillia Center, Haemostasis Unit, Agia Sofia Children's Hospital, Athens, Greece), Prof. Jean Fraoncois Schved and Dr. Muriel Giansily-Blaizot (Hopital Saint Eloi, Montpellier, France, and associated centers).

\section{References}

[1] R. Palla, F. Peyvandi, A.D. Shapiro, Rare bleeding disorders: diagnosis and treatment, Blood 125 (13) (2015) 2052-2061. 
[2] F. Peyvandi, R. Palla, M. Menegatti, S.M. Siboni, S. Halimeh, B. Faeser, et al., Coagulation factor activity and clinical bleeding severity in rare bleeding disorders: results from the European Network of Rare Bleeding Disorders, J. Thromb. Haemost. 10 (4) (2012) 615-621.

[3] J.E. Sadler, Von Willebrand disease type 1: a diagnosis in search of a disease, Blood 101 (6) (2003) 2089-2093.

[4] A.C. Mauer, N.A. Khazanov, N. Levenkova, S. Tian, E.M. Barbour, C. Khalida, et al., Impact of sex, age, race, ethnicity and aspirin use on bleeding symptoms in healthy adults, J. Thromb. Haemost. 9 (1) (2011) 100-108.

[5] J.M. Higham, P.M. O'Brien, R.W. Shaw, Assessment of menstrual blood loss using a pictorial chart, Br. J. Obstet. Gynaecol. 97 (8) (1990) 734-739.

[6] A. Srámek, J.C. Eikenboom, E. Briët, J.P. Vandenbroucke, F.R. Rosendaal, Usefulness of patient interview in bleeding disorders, Arch. Intern. Med. 155 (13) (1995) 1409-1415.

[7] J.E. Sadler, F. Rodeghiero, Provisional criteria for the diagnosis of VWD type 1, J. Thromb. Haemost. 3 (4) (2005) 775-777.

[8] F. Rodeghiero, G. Castaman, A. Tosetto, J. Batlle, F. Baudo, A. Cappelletti, et al., The discriminant power of bleeding history for the diagnosis of type 1 von Willebrand disease: an international, multicenter study, J. Thromb. Haemost. 3 (12) (2005) 2619-2626.

[9] M. Bowman, G. Mundell, J. Grabell, W.M. Hopman, D. Rapson, D. Lillicrap, P. James, Generation and validation of the Condensed MCMDM-1VWD Bleeding Questionnaire for von Willebrand disease, J. Thromb. Haemost. 6 (12) (2008) 2062-2066.

[10] F. Rodeghiero, A. Tosetto, T. Abshire, D.M. Arnold, B. Coller, P. James, et al., ISTH/SSC bleeding assessment tool: a standardized questionnaire and a proposal for a new bleeding score for inherited bleeding disorders, J. Thromb. Haemost. 8 (9) (2010) 2063-2065.

[11] S.M. Siboni, M. Spreafico, L. Calò, A. Maino, E. Santagostino, A.B. Federici, F. Peyvandi, Gynaecological and obstetrical problems in women with different bleeding disorders, Haemophilia 15 (6) (2009) 1291-1299.

[12] A. Kulkarni, C.A. Lee, A. Griffeon, R.A. Kadir, Disorders of menstruation and their effect on the quality of life in women with congenital factor VII deficiency, Haemophilia 12 (3) (2006) 248-252.
[13] S.E. Shapiro, E. Phillips, R.A. Manning, C.V. Morse, S.L. Murden, M.A. Laffan, A.D. Mumford, Clinical phenotype, laboratory features and genotype of 35 patients with heritable dysfibrinogenaemia, Br. J. Haematol. 160 (2) (2013) 220-227.

[14] A. Tosetto, G. Castaman, I. Plug, F. Rodeghiero, J. Eikenboom, Prospective evaluation of the clinical utility of quantitative bleeding severity assessment in patients referred for hemostatic evaluation, J. Thromb. Haemost. 9 (6) (2011) 1143-1148.

[15] H.A.G. Azzam, H.R. Goneim, A.M. El-Saddik, E. Azmy, M. Hassan, S. El-Sharawy, The Condensed MCMDM-1 VWD Bleeding Questionnaire as a predictor of bleeding disorders in women with unexplained menorrhagia, Blood Coagul. Fibrinolysis 23 (4) (2012) 311-315.

[16] R. Earl, Definition of major and minor surgery. A question and an answer, Ann. Surg. 65 (6) (1917) 799.

[17] F. Peyvandi, D. Di Michele, P.H. Bolton-Maggs, C.A. Lee, A. Tripodi, A. Srivastava, Project on consensus definitions in rare bleeeding disorders of the Factor VIII/Factor IX scientific and standardisation Committee of the International Society on Thrombosis and Haemostasis. Classification of rare bleeding disorders (RBDs) based on the association between coagulant factor activity and clinical bleeding severity, J. Thromb. Haemost. 10 (9) (2012) 1938-1943.

[18] A. Tosetto, F. Rodeghiero, G. Castaman, A. Goodeve, A.B. Federici, J. Batlle, et al., A comparison between two semi-quantitative bleeding scales for the diagnosis and assessment of bleeding severity in type 1 von Willebrand disease, Haemophilia 17 (1) (2011) 155-171.

[19] T. Wahlberg, M. Blomback, P. Hall, G. Axelsson, Application of indicators, predictors and diagnostic indices in coagulation disorders. I. Evaluation of a self-administered questionnaire with binary questions, Methods Inf. Med. 19 (4) (1980) 194-200.

[20] A. Tosetto, F. Rodeghiero, G. Castaman, A. Goodeve, A.B. Federici, J. Batlle, et al., A quantitative analysis of bleeding symptoms in type 1 von Willebrand disease: results from a multicenter European study (MCMDM-1 VWD), J. Thromb. Haemost. 4 (4) (2006) 766-773.

[21] M. Daniel, M.C. Jaberoo, R.E. Stead, V.M. Reddy, A.A. Moir, Is admission for epistaxis more common in Caucasian than in Asian people? A preliminary study, Clin. Otolaryngol. 31 (5) (2006) 386-389. 